J Adv Nurs. 2014 Feb;70(2):454-68.

\title{
Development and psychometric testing of the online Adolescent Diabetes Needs Assessment Tool (ADNAT).
}

Cooper H, Spencer J, Lancaster GA, Titman A, Johnson M, Wheeler SL, Lwin R.

Department of Community Health and Well-being, University of Chester, UK; Research and

Development Department, Alder Hey Children's NHS Foundation Trust, Liverpool, UK.

\begin{abstract}
Aim. To report on the development and psychometric testing of the Adolescent Diabetes Needs Assessment Tool.

Background. The UK has the fifth largest paediatric diabetes population in the world, but one of the poorest levels of diabetes control, highlighting the need for intervention development.

Design. Mixed methods following recommendations for questionnaire design and validation.

Methods. A total of 171 young people (12-18 years) participated between 2008-2011. Methods included item selection using secondary framework analysis, item review, pre-testing, piloting and online transfer. Statistical tests assessed reliability using item-total correlations, inter-item consistency and test-retest reliability; and validity using blood glucose (HbA1c) levels and the SelfManagement of type 1 Diabetes in Adolescence questionnaire.

Results. The Adolescent Diabetes Needs Assessment Tool consists of 117 questions divided between six domains of educational and psychosocial support needs. It combines reflective questioning with needs assessment to raise self-awareness to support adolescent decision-making in relation to diabetes self-care. Thirty-six of the questions provide self-care and psychosocial health assessment scores. Face and content validity of the scoring items were all positively evaluated in terms of appropriateness and readability and tests for validity found significant correlations with SelfManagement of type 1 Diabetes in Adolescence and weak correlation with HbA1c, which compared favourably with Self-Management of type 1 Diabetes in Adolescence, the only comparable (USA) tool. Item response analysis validated the use of simple additive scores.

Conclusions. The Adolescent Diabetes Needs Assessment Tool combines reflective learning with needs assessment to support patient-centred clinical consultations.
\end{abstract}

Keywords: Instrument development, needs assessment, nursing, tailored education, technology, type 1 diabetes, young people

PMID: 23998442

\section{Supplement:}

For a young person with type 1 diabetes and their families, much of life is spent managing their health with a focus on achieving glycaemic targets. The role of the paediatric diabetes team is to help them to do this to the best of their ability by matching it with their individual needs. Whilst glucose targets play an important part in treatment, they do not provide information on how a young person is actually coping with their diabetes, or what is important to them at any point in time. This observation is important because a recent (2012-13) UK National Paediatric Diabetes Audit found that only $17 \%$ of young people achieved recommended glycaemic targets, $25 \%$ were at high risk for future complications, and only $12 \%$ had all their care processes recorded. Importantly, the Audit demonstrated that over $90 \%$ of glycaemic variability is due to service related factors including standards of diabetes education and the way it is delivered. This unacceptable picture has remained relatively unchanged over the last 10 years. The UK Government responded in 2012 by introducing a 
paediatric diabetes 'Best Practice Tariff' which specifies tailored education based on personal need as one of 14 mandatory care standards.

Our research programme, which began in 2006, followed the Medical Research Council guidance on developing and evaluating complex interventions. We studied adolescent diabetes self-care, diabetes team working, technological methods of learning, and investigated relevant theory. The developing relevance of technology based education to young people (digital natives) was highlighted by a metaanalysis of 46 studies which showed that a blend of technology enhanced learning and face-to-face instruction had stronger learning outcomes than did face-to-face instruction alone in primary, secondary and tertiary education. At this time, existing diabetes websites were directed primarily toward adults, had wide variations in the quality of evidence provided, and offered didactic information at high reading levels with little interactive technology, social support or problem-solving assistance. Paediatric research in this area was also lacking, both in terms of quantity and quality. Systematic reviews, including our own, consistently highlighted an absence of rigorous UK based research, minimal use of theory, and no reporting of process, health inequalities, dose response and cost-effectiveness data. In addition, findings highlighted the need to personalise learning in alignment with developmental stages i.e. age-related reasoning and cognitive abilities making regular needs assessment (and reassessment) a core requirement. No such instrument was located in the UK and we therefore aimed to fill this gap in service provision by producing ADNAT.

A collaboration of young people, parents and health professionals from six different regions in the North West of England covering wide socio-economic variation provided guidance for the instrument content, language and testing. Focus groups were also run to ensure it met users' needs. The final result was the ADNAT App ( which consists of 117 questions divided between 6 domains including: all about me, physical activity, eating, monitoring blood glucose, medication taking and living with diabetes. Whilst the total number of questions is large, the actual number answered by users is filtered according to, for example, insulin regimen and lifestyle factors. Thirty-six of the questions, 'hidden' amongst the remainder, provide two scored traffic light coloured 'Needs Assessment Ratings' (NARs) to identify those with high (red), medium (amber), and least (green) needs. These scores provide a measure of change over time and can therefore be used to deliver audit information relating to patient self-care education and also professional accountability.

The tool is intended to work in two ways. For patients, it aims to raise self-awareness about their diabetes self-care and how they are coping with their condition through online reflective questioning and instant assessment feedback. For paediatric diabetes practitioners, it provides a patient-centred communication tool that can be used to work collaboratively with their patients by targeting conversation and setting goals according to identified needs. ADNAT therefore acts as the mediator for facilitating tailored education and support. Given its mobile technological format, it has flexibility with respect to where it can be accessed, and it auto-saves allowing patients to complete it at different times, rather than all at once. It has large data storage space making it a resource efficient tool, and has the potential to provide educational audit data through its scoring mechanism. ADNAT also provides 'connected information' making patient information accessible to the multidisciplinary team so that it can reduce multiple collections of data, having the potential to streamline care processes, reduce the burden of paperwork and provide information for accurate 'diagnosis' of individual need. In this way it may reduce unnecessary referrals, promote team working, increase clinical productivity and improve patient satisfaction. 

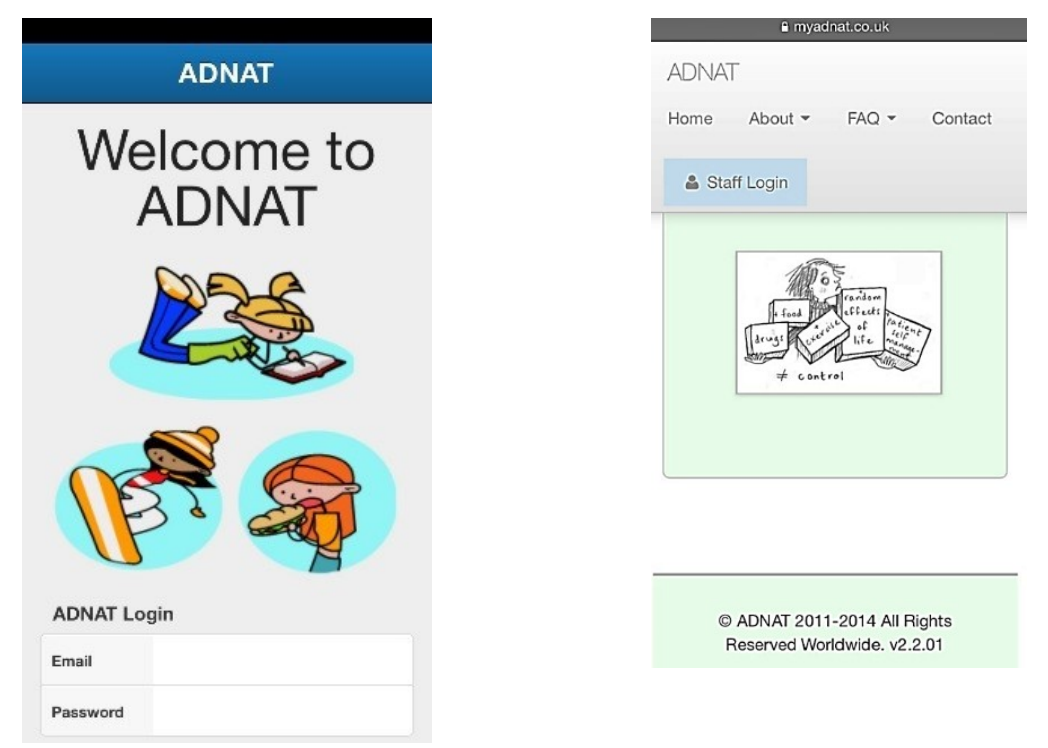

ADNAT has now been included in the UK's National Paediatric Diabetes Improvement Plan for 20132018. However, we are aware that the clinical context in which ADNAT 'sits' is key to its implementation. In the UK, the paediatric diabetes service is undergoing changes and because of this, can be identified as a complex interacting and adaptive system. This ontological approach has underpinned the ADNAT research programme, and continues to do so as we now evaluate its diffusion into three pilot sites. In this study we are researching staff perceptions of ADNAT, and contextual and managerial factors within the organisations which affect its implementation. Results are due to be published in 2015. 